Short communication

\title{
Lactobacillus crispatus represses vaginolysin expression by BV associated Gardnerella vaginalis and reduces cell cytotoxicity
}

\author{
Joana Castro ${ }^{\text {a, b, } 1}$, Ana Paula Martins ${ }^{\text {a, } 1}$, Maria Elisa Rodrigues a , Nuno Cerca ${ }^{\text {a, * }}$ \\ ${ }^{a}$ Centre of Biological Engineering (CEB), Laboratory of Research in Biofilms Rosário Oliveira (LIBRO), University of Minho, Campus de Gualtar, Braga, \\ Portugal \\ b Instituto de Ciências Biomédicas Abel Salazar (ICBAS), University of Porto, Porto, Portugal
}

\section{A R T I C L E I N F O}

\section{Article history:}

Received 26 October 2017

Received in revised form

29 January 2018

Accepted 31 January 2018

Available online 7 February 2018

Handling Editor: Andrew B Onderdonk

Keywords:

Bacterial vaginosis

G. vaginalis

L. crispatus

Vaginolysin

Sialidase

\begin{abstract}
A B S T R A C T
Using a chemically-defined medium simulating genital tract secretions, we have shown that preadhering Lactobacillus crispatus to Hela epithelial cells reduced cytotoxicity caused by Gardnerella vaginalis. This effect was associated to the expression of vaginolysin and was specific to L. crispatus interference, as other vaginal facultative anaerobes had no protective effect.
\end{abstract}

(C) 2018 Elsevier Ltd. All rights reserved.
Lactobacillus crispatus is an important urogenital species that is routinely found in the vagina of healthy women [1,2], contributing to the maintenance of normal vaginal microbiota, while its absence has been associated with a range of vaginal abnormalities, especially bacterial vaginosis (BV) $[3,4]$. BV is the leading vaginal disorder in women of reproductive age worldwide and it has been associated with serious public health consequences, including pelvic inflammatory disease [5], acquisition and transmission of the HIV virus [6] and preterm birth [7]. It has been proposed that Gardnerella vaginalis, a facultative anaerobe, is the pathogen responsible for the initiation of BV [8,9]. This bacteria is capable of adhering to vaginal cells, establish biofilms and induce cytotoxicity on vaginal epithelial cells [10,11]. However, despite being the most prevalent and virulent species found in BV, G. vaginalis can also be a part of the vaginal microbiota in healthy women [12,13]. Genetic differences among $G$. vaginalis strains may underlie the diverse pathological features and outcomes that have been associated with

\footnotetext{
* Corresponding author. Centre of Biological Engineering (CEB) , Laboratory of Research in Biofilms Rosário Oliveira (LIBRO), University of Minho, Campus de Gualtar, 4710-057 Braga, Portugal.

E-mail address: nunocerca@ceb.uminho.pt (N. Cerca).

1 J. C. and A. P. M. contributed equally to this work.
}

this species, raising the possibility that distinct pathogenic and non-pathogenic strains or even subspecies exist [14-18]. The G. vaginalis diversity might represent a critical turning point in clarifying ecological interactions and virulence factors contributing to symptoms and sequelae of BV [19]. Therefore, understanding the interactions between beneficial lactobacilli and G. vaginalis is of extreme importance to help unravel the pathogenesis and progression of this condition.

This study aimed to investigate whether the BV-positive and BVnegative $G$. vaginalis strains differ in their abilities to interact with a cervical epithelial (HeLa) cell monolayer pre-treated with L. crispatus, using a functional cytotoxicity model, which represents a significant improvement over our previous study [10], since we used a medium simulating genital tract secretions (mGTS) [20] and epithelial cells were previously covered with $L$. crispatus, mimicking a normal vaginal ecosystem. First, bacterial suspensions of three BV-positive G. vaginalis strains (UM067, NCBI accession number: KP996675.1; UM121, NCBI: KP996681.1; UM241, NCBI: KP996683.1), three BV-negative G. vaginalis strains (UM016, NCBI: KP996686.1; UM085, NCBI: KP996679.1; UM131, NCBI: KP996676.1) and L. crispatus EX533959VC06 were grown in mGTS for $48 \mathrm{~h}$ at $37^{\circ} \mathrm{C}$ with $10 \% \mathrm{CO}_{2}$ (Shel Lab, Cornelius Oregon, USA) [20]. For the cytotoxicity assays, L. crispatus suspension, adjusted to 
$1 \times 10^{7} \mathrm{cfu} / \mathrm{mL}$, was added to a monolayer of HeLa cells for $3 \mathrm{~h}$. Afterwards, blind bacterial suspensions, adjusted to the same concentration, were added to a HeLa cells monolayer pre-adhered with $L$. crispatus for $3 \mathrm{~h}$. Additional controls were conducted to analyze whether $L$. crispatus effects reflect a specie-dependent response or whether other vaginal associated species can exhibit similar effects. Thus, herein, we included the following bacterial species as controls: Corynebacterium tuberculostearicum UM137Ct2 (NCBI: KT805279); Staphylococcus warnerii UM224Sw (NCBI: KT923488); and Nosocomiicoccus ampullae UM121Na (NCBI: KT805272), due to their low cytotoxicity and adhesion levels similar to L. crispatus [21]. Cytotoxicity was scored on a 0 to 5 scale [11]. Numeric scores were assigned as follows: 0 , no difference between the test and the control; $1,25 \%$ of the cells were rounded; $2,25-50 \%$ of the cells were rounded; $3,50 \%$ of the cells were rounded with partial monolayer disruption; 4, 50\% cells were rounded, with extensive disruption of the monolayer; and 5 , complete disruption or absence of the monolayer.

Furthermore, we also quantified the expression levels of vaginolysin ( $v l y$ ) and sialidase (sld) transcripts in three different conditions: G. vaginalis planktonic cells (pre-infection) (i); after G. vaginalis infection on a monolayer of HeLa cells (post-infection in HeLa) (ii); and after G. vaginalis infection on a monolayer of HeLa cells pre-treated with $L$. crispatus (post-infection in HeLa with Lc) (iii). Briefly, total RNA of these three different conditions was extracted using an ExtractME RNA Bacteria \& Yeast kit (Blirt S.A., Poland) with minor changes, as optimized before [22]. Quantitative PCR (qPCR) was prepared by mixing together $5 \mu \mathrm{L}$ of iQ SYBR green supermix (Bio-Rad, Hercules, CA, USA), $2 \mu \mathrm{L}$ of 1:100 diluted cDNA, $0.5 \mu \mathrm{L}$ of $5 \mu \mathrm{M}$ Forward and Reverse primes and water up to $10 \mu \mathrm{L}$. Primer sequences for target genes are listed in our previous studies, as follows: 16sRNA (Fw and Rv) [23]; vly (Fw2 and Rv2) and sld (Fw1 and Rv1) [10]. Normalized gene expression was determined by using the delta $C_{t}$ method $\left(E^{\Delta C t}\right)$, a variation of the Livak method, where $\Delta C_{t}=C_{t}$ (reference gene) - $C_{t}$ (target gene) and $E$ stands for the reaction efficiency experimentally determined. At least three biologic replicates of each condition were performed. The data were analyzed using the $t$-test or A-NOVA with the statistical software package GraphPad Prism version 6 (GraphPad Software Inc., La Jolla, CA, USA). $P$-values of less than 0.05 were considered significant.

Our results highlighted that BV-positive G. vaginalis strains were able to induce more extensive damages on the HeLa monolayer than BV-negative strains (Fig. 1a), supporting our previous data [10]. Remarkably, when L. crispatus was pre-adhered to HeLa, cytotoxicity effect of all $G$. vaginalis was significantly reduced $(\mathrm{p}<0.05)$. A possible explanation for this fact could be that L. crispatus is blocking $G$. vaginalis adherence [24], indicating that competitive exclusion of this species could be a key role protecting the vagina from invading pathogens [25].

Trying to unravel whether this response was L. crispatus-specific, we pre-coated the HeLa monolayer with C. tuberculostearicum, N. ampullae or S. warnerii. Curiously, we verified that the selected bacterial species were not able to reduce the cytopathogenic alterations caused by $G$. vaginalis on the epithelial monolayer (Fig. 1b). This supports the specific role of $L$. crispatus in reducing G. vaginalis cytotoxicity. Recent studies have shown that cytoprotective effect of $L$. crispatus seems to be related with stimulation of immune response [26], reduction of cell apoptosis [27], or changes on the physical properties of the plasma membrane in HeLa cells [28].

The different cytotoxic activity between BV-negative and BVpositive isolates could be due to a pre-forming toxin produced by G. vaginalis, vaginolysin, which is able to induce cell death and is thus a virulence factor [29]. Furthermore, G. vaginalis virulence has also been associated to sialidase [30]. This enzyme is known to facilitate the destruction of the protective mucus layer on the vaginal epithelium [30]. Therefore, to compare the cytotoxicity effect and expression levels of $v l y$ and sld transcripts between the two groups of strains, we used a BV-positive G. vaginalis UM241 and a BV-negative $G$. vaginalis UM131 strain, which carry both genes of interest (Castro et al., 2015). Our results revealed differences in the expression of both genes, being the transcript levels of $v l y$ (Fig. 2a) higher when compared to the transcript levels of sld (Fig. 2b), similar to what was verified in our previous report [10].

Interestingly, our data also revealed that after post-infection by a)

- G. vaginalis infection on HeLa cells monolayer

- G. vaginalis infection on HeLa cells monolayer pre-treated with L. crispatus

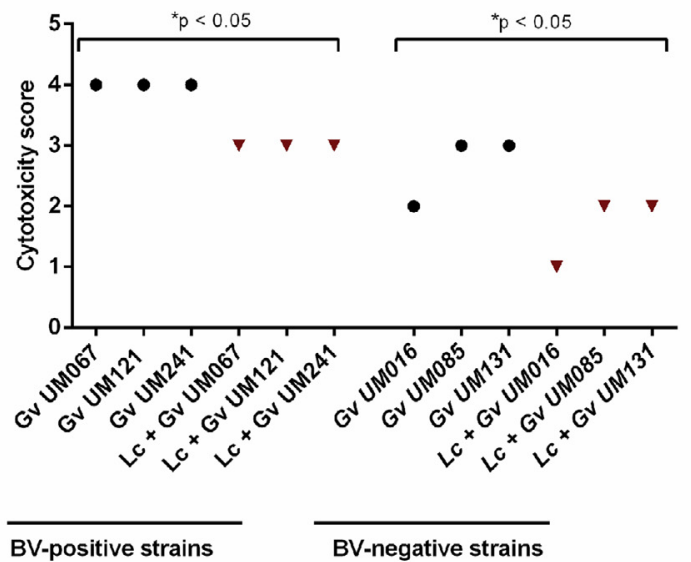

b)
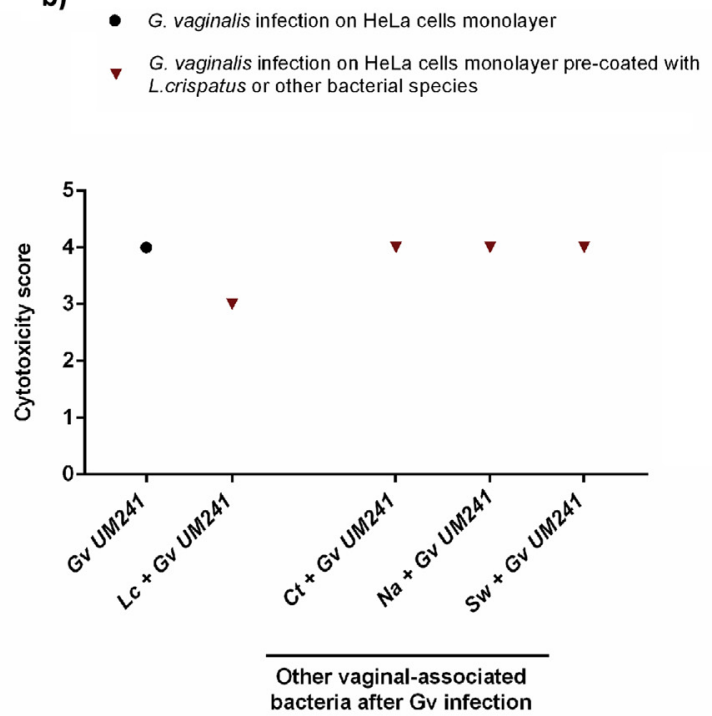

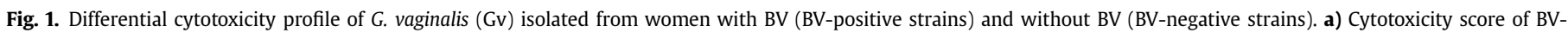

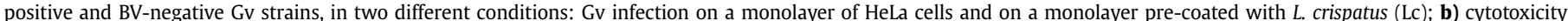

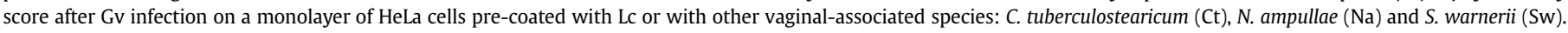
*Values are significantly different between the 2 groups of Gv strains under the same conditions (one-way ANOVA, $\mathrm{p}<0.05$ ). 
a)

Vaginolysin (vly)

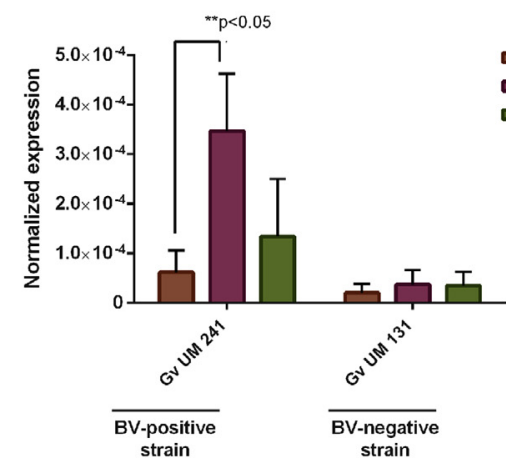

b) Sialidase (s/d)
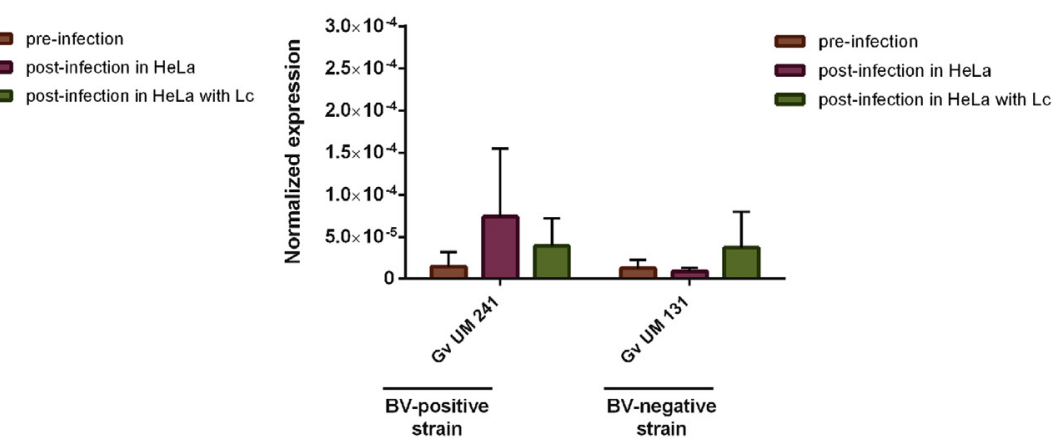

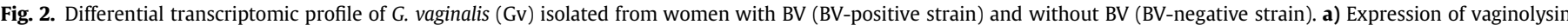

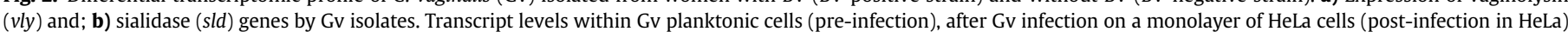

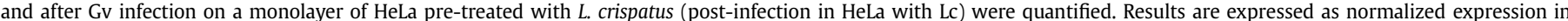

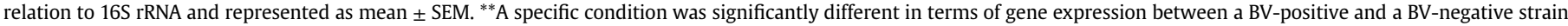
(T-test, $\mathrm{p}<0.05)$.

a BV-positive strain, transcript levels of both genes were significantly higher than in pre-infection (Fig. 2a and b). Remarkably, precoating the HeLa monolayer with $L$. crispatus caused a repression of expression in 2.58-fold and 1.89-fold for vly and sld transcripts, respectively. Regarding the BV-negative strain, the same tendency was observed for vly expression between the post- and preinfection conditions (Fig. 2a). However, no differences were detected in sld expression (Fig. 2b). Surprisingly, our findings suggest that no direct association seems to exist between $v l y$ and sld expression by a BV-negative group and the presence of $L$. crispatus, despite the ability of this species to repress the cytotoxic activity of both G. vaginalis groups.

Taking in consideration our novel findings and our previous observations $[10,24]$ we underline the importance of $L$. crispatus, since it seems to possess some factors that can trigger protective mechanisms against BV-positive G. vaginalis strains [25]. A limitation of our study was the use of only a representative Lactobacillus species, correlated with healthy vaginal microflora, and we did not explore how other lactobacilli would interact with either G. vaginalis or other bacterial species found in BV. However, there is no doubt that a refined genomic characterization of the G. vaginalis strains might allow a better knowledge of the molecular mechanisms behind the different patterns of cytotoxicity.

\section{Acknowledgements}

This work was supported by Portuguese National Funds (FCT) under the scope of the strategic funding of UID/BIO/04469/2013 unit and COMPETE 2020 (POCI-01-0145-FEDER-006684). JC, and MER acknowledge the financial support of individual Grants SFRH/ $\mathrm{BD} / 93963 / 2013$, and SFRH/BPD/95401/2013 respectively. NC is an Investigador FCT.

\section{References}

[1] R.F. Lamont, J.D. Sobel, R.A Akins, S.S Hassan, T. Chaiworapongsa, J.P. Kusanovic, et al., The vaginal microbiome: new information about genital tract flora using molecular based techniques, BJOG 118 (2011) 533-549. https://doi.org/10.1111/j.1471-0528.2010.02840.x.

[2] J. Ravel, P. Gajer, Z. Abdo, G.M. Schneider, S.S. Koenig, S.L. McCulle, et al. Vaginal microbiome of reproductive-age women, Proc. Natl. Acad. Sci. U. S. A. 108 (Suppl 1) (2011) 4680-4687. https://doi.org/10.1073/pnas.1002611107.

[3] J.M. Macklaim, A.D. Fernandes, J.M. Di Bella, J.A. Hammond, G. Reid, G.B. Gloor Comparative meta-RNA-seq of the vaginal microbiota and differential expression by Lactobacillus iners in health and dysbiosis, Microbiome 1 (2013) 12. https://doi.org/10.1186/2049-2618-1-12.
[4] H. Verstraelen, R. Verhelst, G. Claeys, E. De Backer, M. Temmerman, M. Vaneechoutte, Longitudinal analysis of the vaginal microflora in pregnancy suggests that $L$. crispatus promotes the stability of the normal vaginal microflora and that $L$. gasseri and/or $L$. iners are more conducive to the occurrence of abnormal vaginal microflora, BMC Microbiol. 9 (2009) 116. https://doi.org/10.1186/1471-2180-9-116.

[5] H. Sharma, R. Tal, N.A. Clark, J.H. Segars, Microbiota and pelvic inflammatory disease, Semin. Reprod. Med. 32 (2014) 43-49. https://doi.org/10.1055/s0033-1361822.

[6] P. Mirmonsef, L. Krass, A. Landay, G.T. Spear, The role of bacterial vaginosis and trichomonas in HIV transmission across the female genital tract, Curr. HIV Res. 10 (2012) 202-210. PMCID: PMC3788616.

[7] A.S. Dingens, T.S. Fairfortune, S. Reed, C. Mitchell, Bacterial vaginosis and adverse outcomes among full-term infants: a cohort study, BMC Pregnancy Childbirth 16 (2016) 278. https://doi.org/10.1186/s12884-016-1073-y.

[8] J.R. Schwebke, C.A. Muzny, W.E. Josey, Role of Gardnerella vaginalis in the pathogenesis of bacterial vaginosis: a conceptual model, J. Infect. Dis. 210 (2014) 338-343. https://doi.org/10.1093/infdis/jiu089.

[9] A. Machado, N. Cerca, Influence of Biofilm Formation by Gardnerella vaginalis and other anaerobes on bacterial vaginosis, J. Infect. Dis. 212 (2015) 1856-1861. https://doi.org/10.1093/infdis/jiv338.

[10] J. Castro, P. Alves, C. Sousa, T. Cereija, A. Franca, K.K. Jefferson, et al., Using an in-vitro biofilm model to assess the virulence potential of bacterial vaginosis or non-bacterial vaginosis Gardnerella vaginalis isolates, Sci. Rep. 5 (2015) 11640. https://doi.org/10.1038/srep11640.

[11] J.L. Patterson, A. Stull-Lane, P.H. Girerd, K.K. Jefferson, Analysis of adherence, biofilm formation and cytotoxicity suggests a greater virulence potential of Gardnerella vaginalis relative to other bacterial-vaginosis-associated anaerobes, Microbiology 156 (2010) 392-399. https://doi.org/10.1099/mic.0. 034280-0.

[12] A.A. Aroutcheva, J.A. Simoes, K. Behbakht, S. Faro, Gardnerella vaginalis isolated from patients with bacterial vaginosis and from patients with healthy vaginal ecosystems, Clin. Infect. Dis. 33 (2001) 1022-1027. https://doi.org/10. $1086 / 323030$.

[13] R.J. Hickey, L.J. Forney, Gardnerella vaginalis does not always cause bacterial vaginosis, J. Infect. Dis. 210 (2014) 1682-1683. https://doi.org/10.1093/infdis/ jiu303.

[14] C.J. Yeoman, S. Yildirim, S.M. Thomas, A.S. Durkin, M. Torralba, G. Sutton, et al., Comparative genomics of Gardnerella vaginalis strains reveals substantial differences in metabolic and virulence potential, PLoS One 5 (2010), e12411. https://doi.org/10.1371/journal.pone.0012411.

[15] M.D. Harwich Jr., M.G. Serrano, J.M. Fettweis, J.M. Alves, M.A. Reimers, G.A. Buck, et al., Genomic sequence analysis and characterization of Sneathia amnii sp. nov, BMC Genom. 13 (Suppl 8) (2012) S4. https://doi.org/10.1186/ 1471-2164-13-S8-S4.

[16] M. Janulaitiene, V. Paliulyte, S. Grinceviciene, J. Zakareviciene, A. Vladisauskiene, A. Marcinkute, et al., Prevalence and distribution of Gardnerella vaginalis subgroups in women with and without bacterial vaginosis, BMC Infect. Dis. 17 (2017) 394. https://doi.org/10.1186/s12879-017-2501-y.

[17] S.V. Balashov, E. Mordechai, M.E. Adelson, S.E. Gygax, Identification, quantification and subtyping of Gardnerella vaginalis in noncultured clinical vaginal samples by quantitative PCR, J. Med. Microbiol. 63 (2014) 162-175. https:// doi.org/10.1099/jmm.0.066407-0.

[18] J. Castro, N. Cerca, BV and non-BV associated Gardnerella vaginalis establish similar synergistic interactions with other BV-associated microorganisms in dual-species biofilms, Anaerobe 36 (2015) 56-59. https://doi.org/10.1016/j.a 
naerobe.2015.10.008.

[19] J.J. Schellenberg, M.H. Patterson, J.E. Hill, Gardnerella vaginalis diversity and ecology in relation to vaginal symptoms, Res. Microbiol. S0923-2508 (2017) 30060-30068. https://doi.org/10.1016/j.resmic.2017.02.011.

[20] R.L. Stingley, H. Liu, L.B. Mullis, C.A. Elkins, M.E. Hart, Staphylococcus aureus toxic shock syndrome toxin-1 (TSST-1) production and Lactobacillus species growth in a defined medium simulating vaginal secretions, J. Microbiol. Meth. 106 (2014) 57-66. https://doi.org/10.1016/j.mimet.2014.08.002.

[21] P. Alves, J. Castro, C. Sousa, T.B. Cereija, N. Cerca, Gardnerella vaginalis outcompetes 29 other bacterial species isolated from patients with bacterial vaginosis, using in an in vitro biofilm formation model, J. Infect. Dis. 210 (2014) 593-596. https://doi.org/10.1093/infdis/jiu131.

[22] A. França, A.I. Freitas, A.F. Henriques, N. Cerca, Optimizing a qPCR Gene expression quantification assay for $S$. epidermidis biofilms: a comparison between commercial kits and a customized protocol, PLoS One 7 (2012), e37480. https://doi.org/10.1371/journal.pone.0037480.

[23] J. Castro, A. França, K.R. Bradwell, M.G. Serrano, K.K. Jefferson, N. Cerca, Comparative transcriptomic analysis of Gardnerella vaginalis biofilms vs. planktonic cultures using RNA-seq, NPJ Biofilms and Microbiomes 3 (2017) 3. https://doi.org/10.1038/s41522-017-0012-7.

[24] J. Castro, A. Henriques, A. Machado, M. Henriques, K.K. Jefferson, N. Cerca, Reciprocal interference between Lactobacillus spp. and Gardnerella vaginalis on initial adherence to epithelial cells, Int. J. Med. Sci. 10 (2013) 1193. https:// doi.org/10.7150/ijms.6304.
[25] T. Ojala, M. Kankainen, J. Castro, N. Cerca, S. Edelman, B. Westerlund-Wikstrom, et al., Comparative genomics of Lactobacillus crispatus suggests novel mechanisms for the competitive exclusion of Gardnerella vaginalis, B BMC Genomics 15 (2014) 1070. https://doi.org/10.1186/1471-2164-15-1070.

[26] X.X. Niu, T. Li, X. Zhang, S.X. Wang, Z.H. Liu, Lactobacillus crispatus modulates vaginal epithelial cell innate response to Candida albicans, Chin. Med. J. 130 (2017) 273-279. https://doi.org/10.4103/0366-6999.198927.

[27] E. Motevaseli, M. Shirzad, S.M. Akrami, A.S. Mousavi, A. Mirsalehian, M.H. Modarressi, Normal and tumour cervical cells respond differently to vaginal lactobacilli, independent of $\mathrm{pH}$ and lactate, J. Med. Microbiol. 62 (2013) 1065-1072. https://doi.org/10.1099/jmm.0.057521-0.

[28] N. Calonghi, C. Parolin, G. Sartor, L. Verardi, B. Giordani, G. Frisco, et al., Interaction of vaginal Lactobacillus strains with HeLa cells plasma membrane, Benef. Microbes 8 (2017) 625-633. https://doi.org/10.3920/BM2016.0212.

[29] S.E. Gelber, J.L. Aguilar, K.L. Lewis, A.J. Ratner, Functional and phylogenetic characterization of vaginolysin, the human-specific cytolysin from Gardnerella vaginalis, J. Bacteriol. 190 (2008) 3896-3903. https://doi.org/10.1128/JB. 01965-07.

[30] L. Hardy, V. Jespers, M. Van den Bulck, J. Buyze, L. Mwambarangwe, V. Musengamana, et al., The presence of the putative Gardnerella vaginalis sialidase A gene in vaginal specimens is associated with bacterial vaginosis biofilm, PLoS One 12 (2017), e0172522. https://doi.org/10.1371/journal.pone. 0172522. 